\title{
Electromagnetic Energy Propagation in Power Electronic Converters: Toward Future Electromagnetic Integration
}

\author{
JAN ABRAHAM FERREIRA, MEMBER, IEEE, AND J. DAAN VAN WYK, FELLOW, IEEE
}

\author{
Invited Paper
}

\begin{abstract}
Historically, it came about that the analysis and design of the power electronic converter center around currents and voltages in the circuits. During the last decade, the electromagnetic character of power electronic converters became more important due to the noise that the switches generates and electromagnetic interference (EMI) and electromagnetic compatibility (EMC) standards have been put in place to control the electromagnetic noise levels. In this contribution, it is argued that coming to grips with the electromagnetic operation of switch-mode power conversion presents a barrier of knowledge that will have to be crossed in order to achieve future meaningful innovation in switch-mode power conversion. Rigorous electromagnetic analysis using realistic parameters are used to indicate important issues relating fundamental limits and technological paths for future generations of power electronic converters. Electromagnetic integration in three dimensions creates new possibilities to increase power densities.
\end{abstract}

Keywords-Electromagnetic analysis, energy conversion, power electronics, switched-mode power supplies.

\section{Power Electronics Toward Electromagnetic ENERGY PROCESSING}

\section{A. Shrinking Dimensions and Increasing Frequency}

Studying the historical development of power electronics [1], [2] indicates that for a long time after its inception, a discrete, decoupled approach to modeling, design, and construction of power electronic converters was totally adequate. Electrical, thermal, and mechanical modeling and design was decoupled, while the sequential type of manufacturing in use was based on using separate components and after laying them out on a plane, wiring them together.

Manuscript received October 19, 2000; revised December 15, 2000.

J. A. Ferreira is with the Technical University of Delft, 2628 CD Delft, The Netherlands.

J. D. van Wyk is with the Bradley Department of Electrical and Computer Engineering, Virginia Polytechnic Institute and State University, Blacksburg, VA 24061 USA.

Publisher Item Identifier S 0018-9219(01)04104-4.
This approach had a convenient, almost one-to-one correspondence to the lumped element circuit modeling, using ideal component transfer functions. This discrete, sequential thinking has permeated research, development, design methodology, and teaching to the present. Even hybrid, integrated power electronics modules are still constructed in a quasi-discrete way. However, continuing change in power device technology, a continuing increase in power density and/or frequency [3], [4] over the past decades have resulted in close electromagnetic, thermal, and mechanical coupling of all the "components" and interconnections that comprise the converter, accentuating undesirable side effects due to the close proximity of "components," materials used, interconnections necessary, etc. ("parasitics"). As the tendency toward integration of modules for the future continues, the integrated power processor is approaching (see Fig. 1), signaling the necessity for change in the present methodology for analysis and design.

\section{B. The Integrated Power Processor as Electromagnetic Device}

At present, a power electronic converter may be characterized as a spread-out electromagnetic device. The quest for increased power density continues the reduction in dimensions and the tendency toward physical integration. This will eventually make the electromagnetic coupling between different subsections of the converter, the electromagnetic nature of inter- and intraconnections, and the electromagnetic nature of the switching processes dominant for determining the energy processing transfer function. For this eventual electromagnetic energy processor, it follows that the electromagnetic limits will be dominant and will have to be explored. As an aid to analyze this development of power electronics technology, the different fundamental functions performed in a converter had been defined in previous work [5]. This analysis did not include the thermal and mechanical functions and 


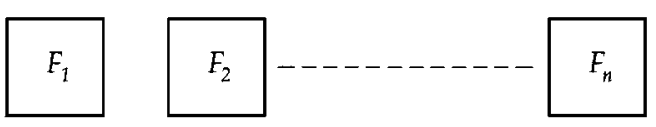

Discrete construction: decoupled transfer functions
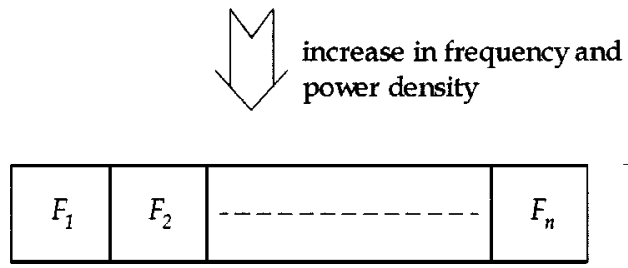

Present quasi-discrete construction: coupled electromagnetic transfer functions

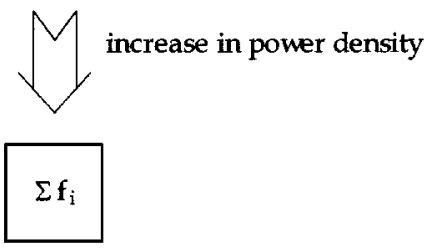

Electromatically integrated power processor, integrated functionality

Fig. 1. Future power electronics integration.

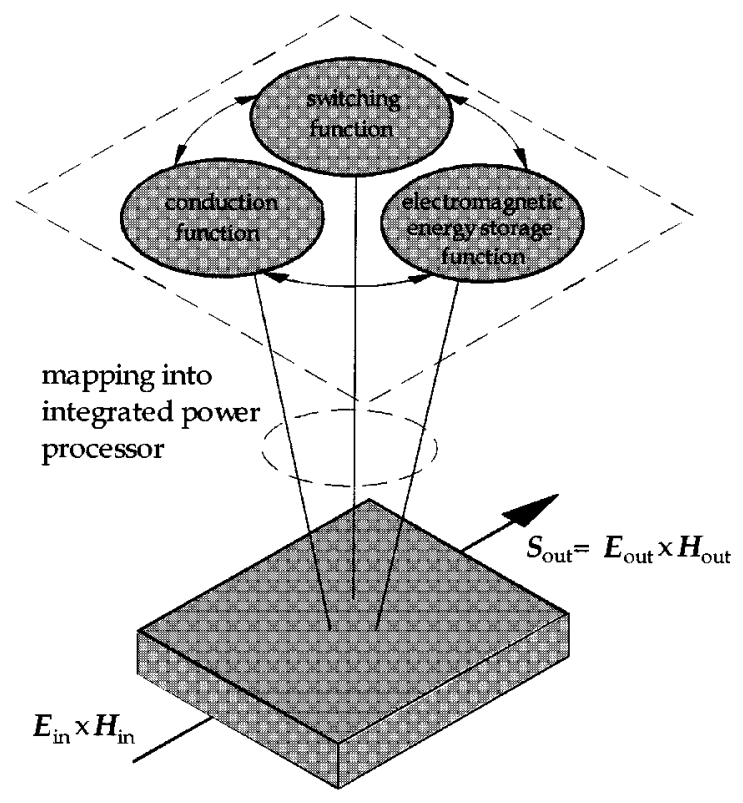

Fig. 2. Future electromagnetic integration paradigm.

these are also being excluded from treatment in this paper. To a first approximation, only the conduction, switching, and electromagnetic energy storage functions will be analyzed here in the way that they map onto converter technology in order to achieve three-dimensional (3-D) energy processing, eventually resulting in an electromagnetically integrated processor (see Fig. 2).

This paper attempts to formulate an initial approach to this future problem by examining the propagation of electromagnetic energy in a power electronic converter. Toward

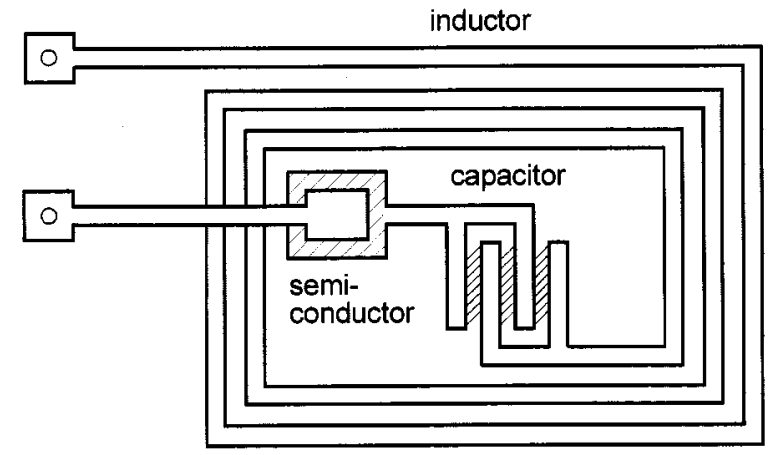

Fig. 3. A simple illustration of a possible physical circuit arrangement inside a module.

this goal, the limitations of circuit theory (Section II), the modeling of electromagnetic energy flow (Section III), electromagnetic energy flux density and the limits thereof (due to materials) (Sections IV and V) are discussed. Electromagnetic energy flux in its relationship to semiconductor switches, capacitive and inductive components, and integrated capacitive-inductive-transformer structures (Section VI) leads to a view of the energy propagation around these structures. Semiconductor switches themselves are in the limit affected by the electromagnetic energy stored in and around them. Eventual structural effects as limits to switching frequencies are formulated from these concepts (Section VII). From the discussions in (Sections III-VII), the energy propagation in a typical converter is examined (Section VIII), and some conclusions are drawn for future electromagnetic integration (Section IX).

\section{LIMITATIONS OF CIRCUIT THEORY}

Circuit theory has evolved into a very powerful modeling tool for electrical circuits. In terms of analogous circuits, it has also become a means to analyze dynamics in system models other than electrical circuits, such as for mechanical and hydraulic systems, leading to the development of much more general network theory. The simulation and modeling tools developed around network theory are in essence toolboxes for solving differential equations. In electrical circuits, a physical meaning can be connected to the constants $R$, $L$, and $C$ under constraints that have achieved somewhat unqualified general acceptance. Resistors, inductors, and capacitors modeled as concentrated elements approximate these very well at low frequencies. At high frequencies, more complicated models comprising subnetworks become necessary and the one-to-one relationship is lost. Additional elements $R, L$, and $C$ are included in the component network, and they should preferably correspond to physical parts of the component structures such as the lead inductance for example. In practice, this is not always possible, especially when cross-coupling effects between the many parts of a subcircuit become important. Eventually this type of modeling degenerates into a sophisticated "curve-fitting" exercise.

In Fig. 3, a simplified two-dimensional (2-D) physical representation of a circuit comprising a capacitor, inductor, and 


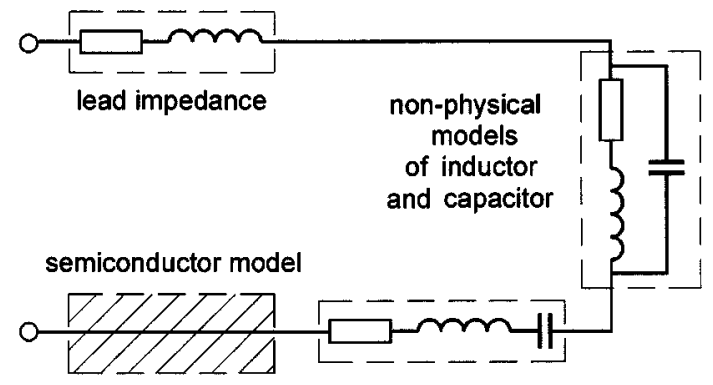

Fig. 4. Lumped element modeling of the physical arrangement of Fig. 4 at higher frequencies.

semiconductor device is used to illustrate how an equivalent high-frequency circuit is extracted for a circuit simulation, as shown in Fig. 4 under the assumptions valid for building a model with lumped elements. In this example, the space in the center of the air-cored spiral winding inductor is used to locate the semiconductor device and capacitor in order to increase the packing density of the module, tending toward hybrid integration. The simplified model shown in Fig. 4, however, totally neglects the increased electromagnetic coupling between "semiconductor device," "capacitor," and "inductor," which in terms of the lumped element modeling technique will use inductive couplings and capacitors to model the effects. However, the following is true:

- it is not possible to separate the component models from the circuit models anymore;

- the models are related to the geometrical design variables of both "components" and "circuit";

- dense packing enhances the parasitics and mutual coupling between different parts of the circuit;

- the influence of eddy currents and other magnetic field related effects become an issue if configurations are used where devices are placed in regions of high magnetic field intensity.

If this packaging technique is developed toward 3-D packaging, the "equivalent circuits" also become 3-D. Consequently elaborate circuit models are needed to analyze the behavior of these types of switch-mode converters. Modern computers are powerful enough to analyze these much more complex modules in terms of networks, but it becomes ever more difficult to interpret the results. If the variation of the electromagnetic characteristics of the materials involved with frequency has to be taken into account additionally, it appears necessary to attempt to approach the problem from an electromagnetic field viewpoint that may add to the insight into the behavior and limitations of these systems.

\section{Modeling EleCtromagnetic EnERGy Flow}

Electromagnetic theory provides two approaches for modeling an electrical phenomenon. The most commonly used approach analyzes currents and voltages and is the backbone of present-day modeling techniques as illustrated in the previous section. The other equally valid method uses electromagnetic fields. Heaviside [6] summarized the analogy between the two methods appropriately as follows.
"Now in Maxell's theory there is the potential energy of the displacement produced in the dielectric parts by the electric force, and there is a kinetic or magnetic induction due to the magnetic force in all parts of the field, including the conductive parts. They are supposed to be set up by the current in the wire. We reverse this: the current in the wire is set up by the energy transmitted through the medium around it."

The general electromagnetic equation for describing the rate of storage and dissipation of energy inside an arbitrary volume is given by the following well-known equation that appears in almost every undergraduate textbook on electromagnetics:

$\oiint(\mathbf{E} \times \mathbf{H}) \cdot d \mathbf{A}=-\oiint\left(\frac{\partial\left(\frac{\varepsilon \mathbf{E}^{2}}{\mathbf{2}}+\frac{\mu \mathbf{H}^{2}}{\mathbf{2}}\right)}{\partial \mathbf{t}}+\mathbf{J} \cdot \mathbf{E}\right) d V$

where

E electric field intensity $(\mathrm{V} / \mathrm{m})$;

H magnetic field intensity $(\mathrm{A} / \mathrm{m})$;

J current density $\left(\mathrm{A} / \mathrm{m}^{2}\right)$;

$\varepsilon \quad$ permittivity $(\mathrm{F} / \mathrm{m})$;

$\mu$ permeability $(\mathrm{H} / \mathrm{m})$.

The first term of the above equation represents the electromagnetic energy that crosses the surface of the volume and the vector cross product of the electric and magnetic fields gives the energy flux vector, commonly known as the Poynting vector [7]

$$
\mathbf{S}=\mathbf{E} \times \mathbf{H}
$$

The Poynting vector has the unit of $\mathrm{W} / \mathrm{m}^{2}$ and can be used to map 3-D energy flux. Electromagnetic energy flux is an alternative to a current and voltage description of circuit behavior. However, the energy flux vector was not unique [8] and different vectors could be devised creating very different pictures of how energy flows in electrical circuits [9]. It was only as recent as 1964 [10] that it was proven that the Poynting vector is the only valid solution for energy flux. Despite attempts to encourage its use [11], this method never became part of formal electrical analysis theory because circuit theory was already entrenched as the preferred approach for analysis. While circuit theory accompanied by modern network simulators has become a very powerful modeling tool, it gives little insight into the processes or mechanisms of energy exchange.

Under the quasi-static conditions that prevail in most power electronic converters, two components of the Poynting vector are relevant. One is due to a charge-induced electric field and the other is due to a magnetically induced electric field

$$
\mathbf{S}=\mathbf{S}_{\text {charge }}+\mathbf{S}_{\text {induced }}
$$

where

$$
\mathbf{S}_{\text {charge }}=-\nabla V \times \mathbf{H}
$$




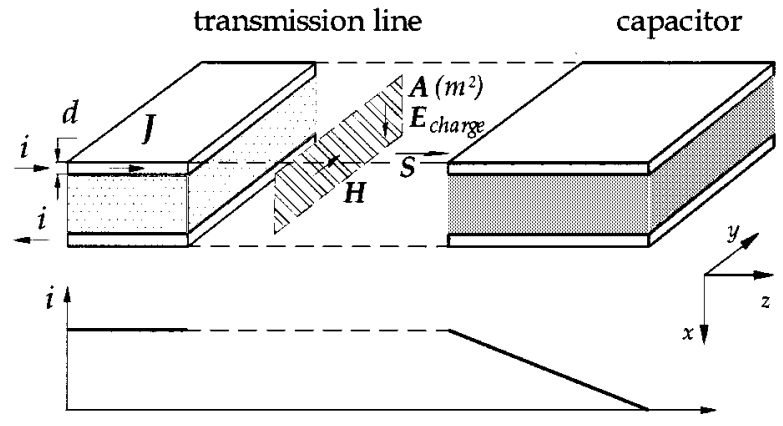

Fig. 5. Energy flux due to charge distribution in planar conductors.

$$
\mathbf{S}_{\text {charge }}=-\frac{\partial \mathbf{A}}{\partial t} \times \mathbf{H}
$$

and $V$ is the electric scalar potential $(\mathrm{V})$ and $\mathbf{A}$ is the magnetic vector potential $(\mathrm{T} \cdot \mathrm{m})$.

If the energy flux is mapped one gets a clear picture of energy flowing in the space between conductors from the source. The energy transfer can be traced, not only to the individual components, but also to the exact spot inside the components where it is converted into another nonelectrical form such as in the electrolyte of a battery, in the slots and windings in machines [12], [13] or into losses in a power electronic converter.

\section{Electromagnetic ENERgy Flux Density and POWER Density in Planar Structures}

\section{A. Energy Flux and Storage Associated with Electrical Charge}

The electrical charge-related energy flux described in (4) is found in conductive structures such as wire or PCB interconnects between components and capacitors. In Fig. 5, a simplified planar representation is shown of an interconnection or power transmission line and capacitive element as encountered for example in the structures of power electronic converters. A transverse electromagnetic energy flux is present between the two conductor plates and propagates in the $z$ direction. In both structures, losses occur and some of the energy flux is converted in to heat. In the case of the capacitor, most of the energy flux is converted into electric field energy. Assuming a periodic function in time, the transverse energy flux can be written as follows:

$$
S_{\text {charge- } z}(\omega t)=E_{x}(\omega t) H_{y}(\omega t) .
$$

If the electric and magnetic fields are described by the following functions:

$$
\begin{aligned}
& H_{y}(\omega t)=J_{0} \cdot d \cdot G(\omega t) \\
& E_{x}(\omega t)=E_{0} \cdot F(\omega t)
\end{aligned}
$$

where $E_{0}$ and $J_{0}$ are the maximum electric field strength and current density, respectively, and $F(\omega t)$ and $G(\omega t)$ are periodic functions with a maximum amplitude of one, then (6) can then be rewritten as follows:

$$
S_{\text {charge- } z}(\omega t)=\hat{S}_{\text {charge }} \cdot F(\omega t) G(\omega t)
$$

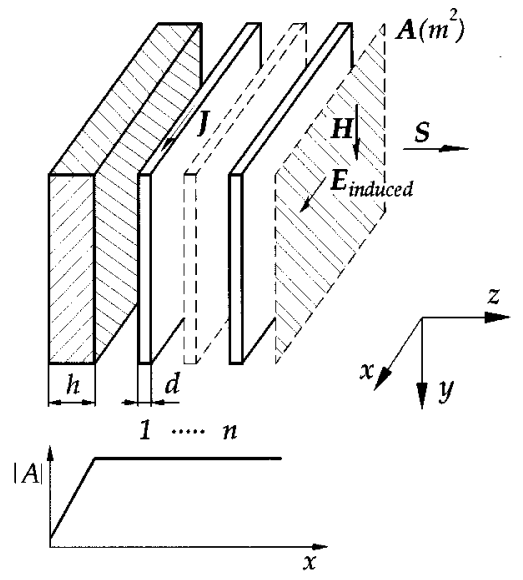

Fig. 6. Energy flux due to magnetic induction.

where the maximum instantaneous electromagnetic energy flux density due to electric charge is given by

$$
\hat{S}_{\text {charge }}=E_{0} \cdot J_{0} \cdot d
$$

The first term of the right-hand expression in (1) represents the rate at which the stored electrical energy changes in the volume. The open-ended portion of the planar structure shown in Fig. 5 will store electrical energy because it functions as a capacitor. This energy is set up by the incident electromagnetic energy flux described by (10). Under conditions of sinusoidal excitation, the electric field energy exchange per second per unit volume can be expressed as

$$
Q_{\text {charge }}=\frac{\omega \varepsilon E_{0}^{2}}{2} .
$$

The energy exchange, as defined in (11) and (17), gives an indication of how much power can be converted if all the energy is buffered in a capacitor or an inductor by charging and discharging the energy storage device fully within every switching cycle. The unit of $Q$ is $\mathrm{W} / \mathrm{m}^{3}$ or $\mathrm{VA} / \mathrm{m}^{3}$ depending on the circuit conditions.

\section{B. Energy Flux and Storage Associated with Magnetic Induction}

Magnetically induced energy flux is found in components that use Faraday's induction principle for their operation. These include inductors, transformers and electrical machines. The transverse electromagnetic energy flux due to magnetic induction can be written as follows:

$$
H_{y}(\omega t)=n \cdot J_{0} \cdot d \cdot G(\omega t)
$$

Fig. 6 shows a planar arrangement to set up a magnetically induced energy flux. On the left-hand side, a magnetic core with a thickness $h$, excited by a magnetic flux density $B$, sets up a magnetic vector potential $A$ that has a constant amplitude on the right-hand side of the core. Implicit to this configuration is a flux return path that exists somewhere to the right of this arrangement. The magnetic vector potential sets 
up an induced electric field given by the following expression:

$$
E_{x}(\omega t)=\omega \cdot B_{0} \cdot h \cdot F(\omega t)
$$

where $B_{0}$ is the maximum flux density (T).

A stack of planar conductors sets up a magnetic field given by the following equation:

$$
H_{y}(\omega t)=n \cdot J_{0} \cdot d \cdot G(\omega t)
$$

where $n$ is the number of current carrying conductors.

Substituting (13) and (14) into (12) gives

$$
S_{\text {induction- } z}(\omega t)=\hat{S}_{\text {induction }} \cdot F(\omega t) G(\omega t)
$$

where the maximum instantaneous magnetically induced electromagnetic energy flux density is given by

$$
\hat{S}_{\text {induction }}=\omega \cdot B_{0} \cdot J_{0} \cdot n \cdot h \cdot d .
$$

The second term of the right-hand expression in (1) represents the rate at which the stored magnetic energy changes in the volume. In magnetic components, the larger part of the energy is stored in air gaps, whether it be localized such as in a gapped inductor or diffuse such as in a powdered iron core. The magnetic energy exchange per second per unit volume can thus be expressed as

$$
Q_{\text {induced }}=\frac{\omega \mu_{0} \mu_{\mathrm{r}}\left(n \cdot d \cdot J_{0}\right)^{2}}{2} .
$$

The above magnetic field energy is not applicable to the core limb on the left-hand side in Fig. 6. The core limb would typically be constructed from a highly permeable core material and that will consequently store little energy. Equation (17) represents the stored energy if the magnetic induced energy flux is fed into an air gap or low permeability core limb on the right-hand side of the arrangement in Fig. 6.

\section{VAlues AND Limits of Electromagnetic FluX DENSITY AND POWER DENSITY DUE TO MATERIALS}

\section{A. Skin Effect in Conductors}

The fact that current only flows on the surface of conductors at high frequencies is one of the most restrictive boundary conditions for power processing. The resistance may actually become larger with increasing wire diameter due to induced eddy currents [14]. Therefore, the thickness of conductors should not exceed the skin depth in practical designs. In planar circuit arrangements, this boundary condition means that thin conducting foil must be used at high frequencies. The foil thickness as a function of frequency is indicated in Fig. 7. One method to get around this restriction is to use very thin copper strands that are isolated from each other, woven, and then connected in parallel. This results in the so-called litz wire, which has been used since the beginning of the century in high-frequency applications such as radio coils [15] but is difficult to realize in planar structures.

In the case of a large solid conductor, the electromagnetic energy flux cannot penetrate the conductor and it is de-

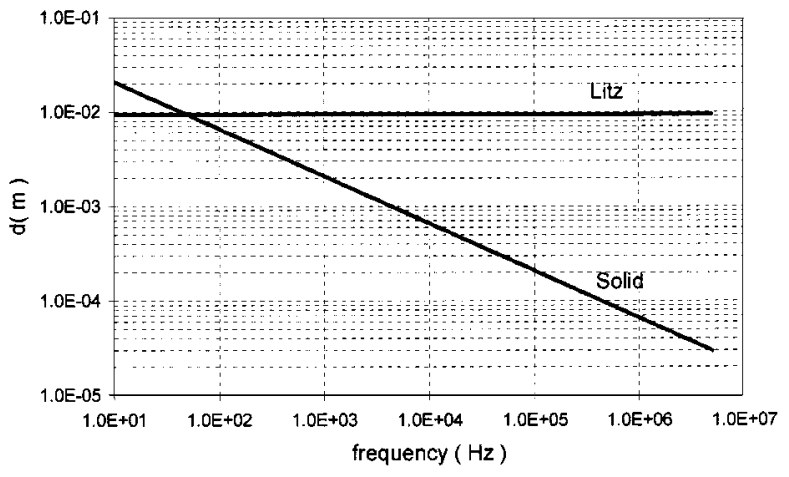

Fig. 7. Conductor thickness equal to skin depth for solid conductors and constant in case of litz wire.

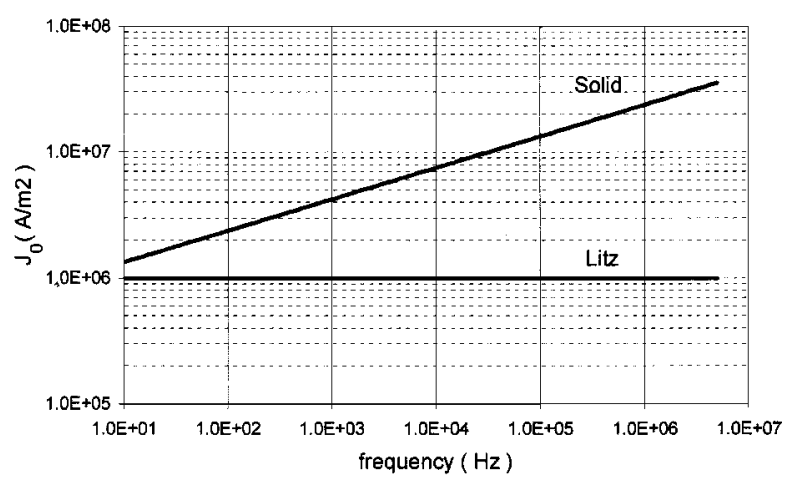

Fig. 8. Current density for constant heat dissipation on conductor surface.

flected around the conductor by the induced eddy currents. Litz wire, however, becomes transparent to electromagnetic energy flux and the diameter of the litz wire bundle is consequently not restricted. For the purpose of the numerical examples in this section, a litz bundle thickness equal to the skin depth at $50 \mathrm{~Hz}$ has been chosen and it is unaffected by the operating frequency, as indicated by the appropriate line in Fig. 7.

As a result of the required insulation and the additional space required for weaving the strands, the effective conductor cross section is reduced and consequently the average current density is affected. In the numerical examples, the average current density is assumed to be half the value used for a solid conductor. In the case of foil conductors, a constant heat dissipation on the surface area is assumed. Based on a current density of $2 \mathrm{~A} / \mathrm{mm}^{2}$ at $50 \mathrm{~Hz}$, the current density as function of frequency is given in Fig. 8, with the frequency-independent behavior of litz conductors again indicated.

\section{B. Electrical and Magnetic Parameters}

The maximum limits of electromagnetic flux density and electromagnetic energy storage depend on the electromagnetic properties of the materials used. The parameters depend on the state of the art of the materials technology. Usually, the electromagnetic excitation of the material needs to be derated as function of frequency. In order to evaluate the limits of energy flux and energy density, assumptions regarding the electromagnetic parameters have to be made. The current den- 


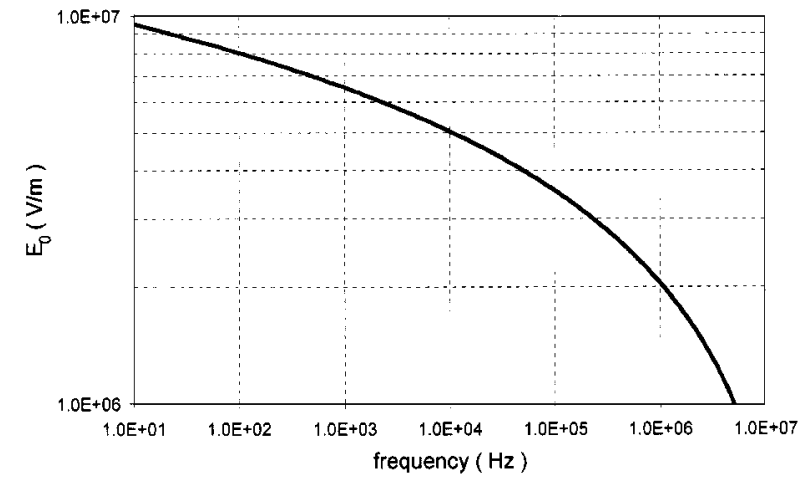

Fig. 9. Derating of electrical field strength as function of frequency.

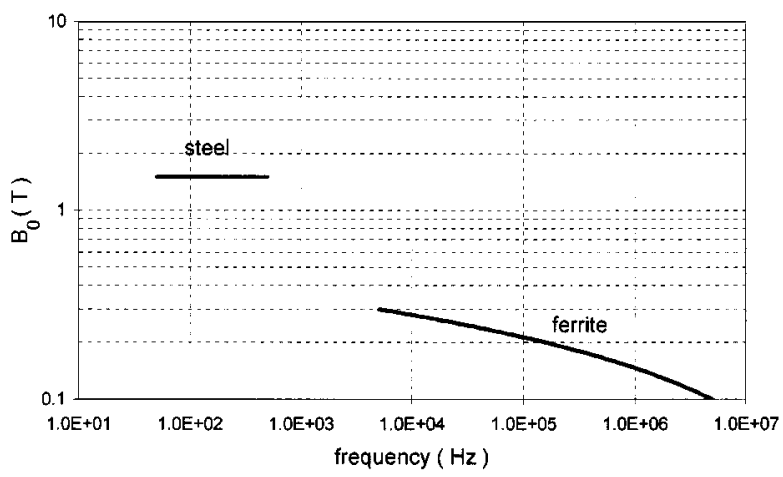

Fig. 10. Flux density: silicon steel at low frequencies and ferrite at high frequencies.

sity assumptions have already been discussed. The value of the electric field intensity as a function of frequency is indicated in Fig. 9 and corresponds to typical values possible with polymer dielectrics.

The most commonly used magnetic material at low frequencies is silicon steel, while ferrites are used at high frequencies. The frequency band is being split using different values of flux density for the two types of material, as shown in Fig. 10. Due to losses, the flux density in ferrite has to be derated as frequency increases. Magnetic material occupies considerably more volume than dielectric material, and the thickness of magnetic cores needs to be restricted due to cost and thermal reasons. Since the heat conductivity of ferromagnetic material in general and silicon steel more specifically is better than ferrite material, the magnetic limbs can be much thicker on the metal cores. The magnetic core limb thickness assumed for the calculations, and as applicable to the planar arrangement shown in Fig. 8, is indicated in the graph of Fig. 11. The usage of conventional-type ferrite cores is assumed up to $500 \mathrm{kHz}$, and from $500 \mathrm{kHz}$ upward microelectronics-based technologies are assumed that limit the thickness of magnetic material to about $100 \mu \mathrm{m}$. The relatively thin cores are due to the low deposition rates of the microelectronic techniques, for example, the sputtering process.

\section{Theoretical Limits of Electromagnetic Energy Flux}

The maximum instantaneous values of electromagnetic energy flux as calculated with (10) and (16) based on all the

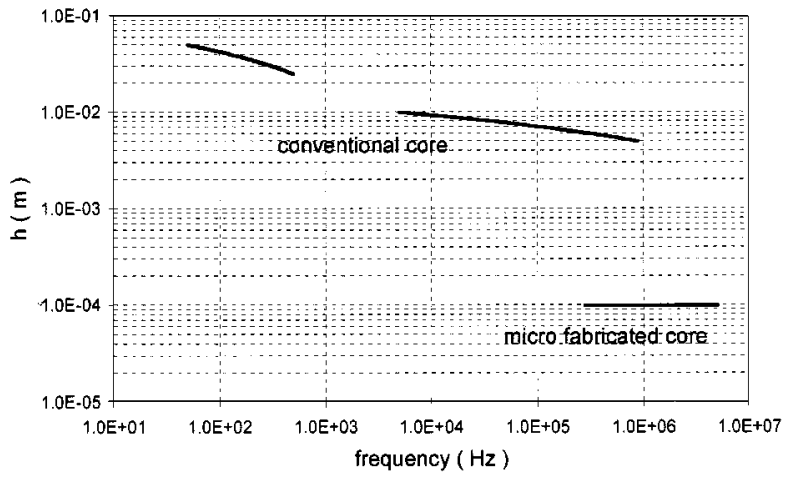

Fig. 11. Magnetic core thickness in three stages: steel core, conventional ferrite core and micro-cores.

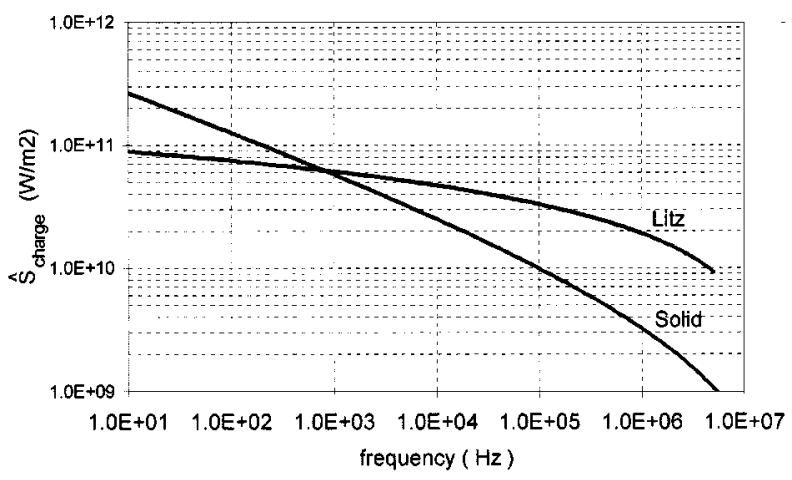

Fig. 12. Maximum electrical charge related energy flux density.

previous assumptions are displayed graphically as a function of frequency in Figs. 12 and 13. A number of observations can be made from these graphs.

- The value of charge-associated energy flux in Fig. 12 is larger than the magnetic induction energy flux in Fig. 13 by orders of magnitude. It should not come as a surprise if one bears in mind that power is usually transmitted between very tightly spaced conductors in circuits while large interface areas exist between the source coil and its load in magnetic components. Even higher values of charge-associated energy flux is possible when superconductors are used in HVDC systems and current densities of $70000 \mathrm{~A} / \mathrm{mm}^{2}$ are used, and energy flux densities of the order of $10^{15} \mathrm{~W} / \mathrm{m}^{2}$ can be achieved.

- The value of flux density of litz wire is lower at low frequencies but surpasses the solid conductors at higher frequencies since it is assumed that the number of strands are increased to compensate for the reduction of strand diameter as the skin depth becomes smaller at high frequencies. However, the difficulties experienced when having to deal with a large number of finely divided strands makes it often impractical.

- The maximum values of charge associated energy flux decreases with frequency while the magnetic-induced energy flux values increase. In practice, it means that the relative volume of interconnections in power electronic circuits that operate at higher frequencies become larger while the transformers become smaller. 


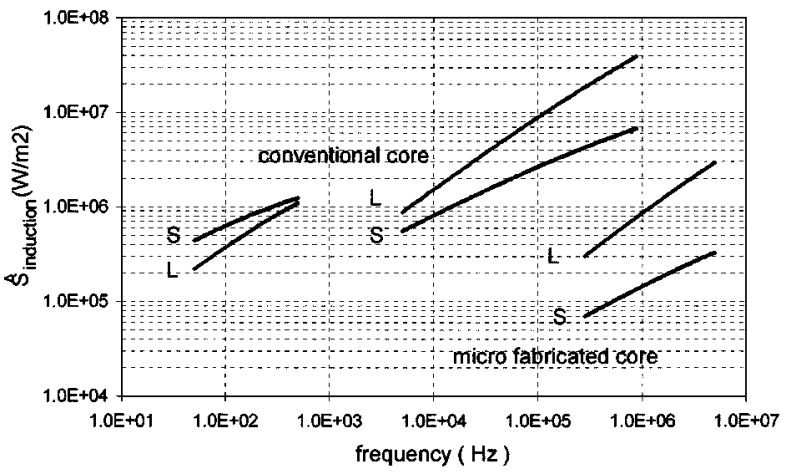

Fig. 13. Maximum magnetically induced energy flux density (litz wire indicated by $L$ and solid wire by $S$ ).

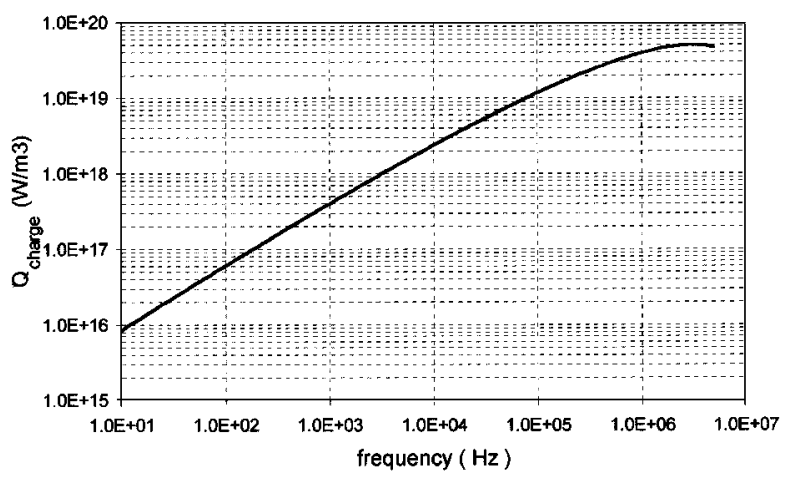

Fig. 14. Maximum electric field energy storage exchange capability with $\varepsilon_{r}=3$.

- The thin layers of magnetic material, made with microelectronic fabrication technologies, makes it difficult to achieve high values of energy flux. As can be seen from (16), the energy flux is independent of the permeability and the low value of energy flux can be attributed to the fact that it is proportional to the thickness of magnetic layer, which is restricted by the process technologies.

\section{Theoretical Limits of Electromagnetic Energy Exchange Density}

The maximum values of electrical and magnetic energy exchange as calculated with (11) and (17), respectively, are displayed graphically as function of frequency in Figs. 14 and 15 . The values increase steadily with frequency, as one would expect, resulting in better power density in switch-mode converters at higher frequencies.

Silicon steel has an advantage over ferrites due to the fact that the flux density is five times larger. However, since ferrites are used in a higher frequency band, the magnetic energy exchange density becomes larger than silicon steel (see Fig. 15), despite the lower flux density. The improvement, however, is not significant in the lower range of the ferrite frequency band. However, the microelectronic fabricated magnetic cores fall severely short as compared to conventional cores.

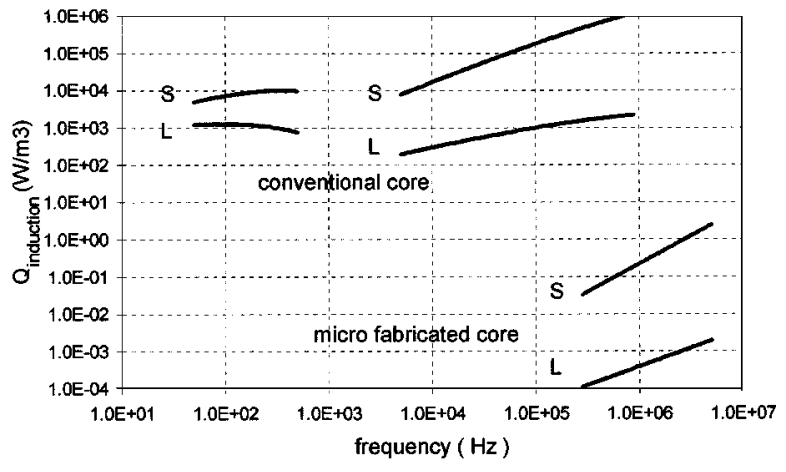

Fig. 15. Maximum magnetic field energy storage capability with $\mu_{r}=3$ (litz wire indicated by $L$ and solid wire by $S$ ).

\section{EleCtromagnetic ENERGy Flow In COMPONENTS}

If electromagnetic energy flow is analyzed in three dimensions, one would get a clear picture of power conversion in three dimensions. Conductors guide and shape the energy flux that flows at velocities close to the speed of light in the space between the wires or conductor strips that carry the go-return current. This is easier said than done, despite the impressive advances in numerical electromagnetic simulation tools. Electromagnetic energy flow requires a full 3-D analysis that merges the quasi-electrostatic and quasi-magnetostatic solutions. One also will have to deal with the superposition of more than one energy flow mechanisms that occur at the same time.

The simple, analytical approach followed in the previous paragraph does not give us the full picture. This will have to wait until suitable electromagnetic analysis tools have been developed. However, it is possible to identify some of the energy flow components in the structures of components and circuits.

\section{A. Semiconductor Switches}

The semiconductor switches modulate the energy flux in time, and although the switch assembly occupies a fairly large volume of the converter, very little energy flux is present inside and around these devices. The reason is that they act as switches and carry either current or voltage, but not both at the same time. Therefore, the vector cross product of electric and magnetic field is small resulting in a small value of the Poynting vector. In the case of ideal switches, they handle no energy flux at all. The role of switches and diodes is thus small in terms of the overall energy flow picture. However, this does not mean that electromagnetic analysis is unimportant for switches. On the contrary, during the switching transient stored magnetic and electric energy has to be moved in and out of the switch assembly, and this causes losses and extra electrical stresses in the silicon chips. This topic will be discussed in detail in a following section.

\section{B. Capacitive Components}

The analysis presented in the previous section calculated the highest energy flux that can be attained. This is one factor 


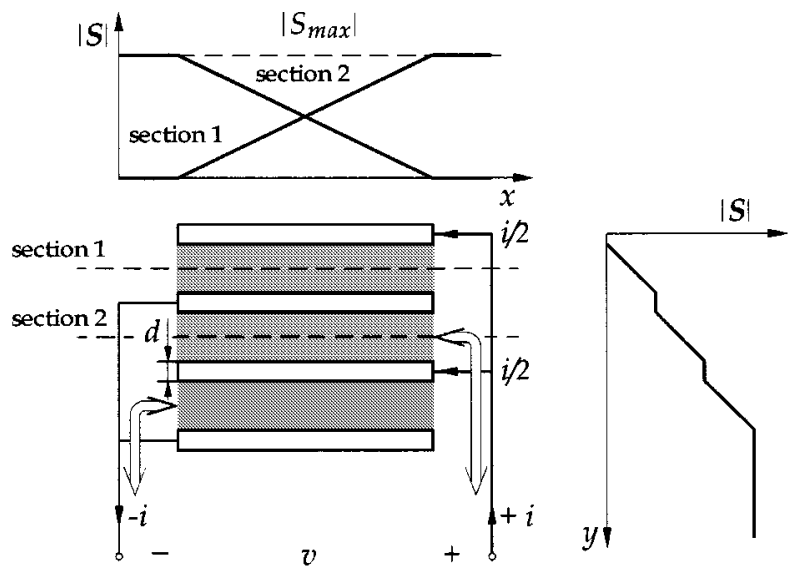

Fig. 16. 2-D multilayer capacitor (electromagnetic energy flux associated with charge indicated by a clear arrow).

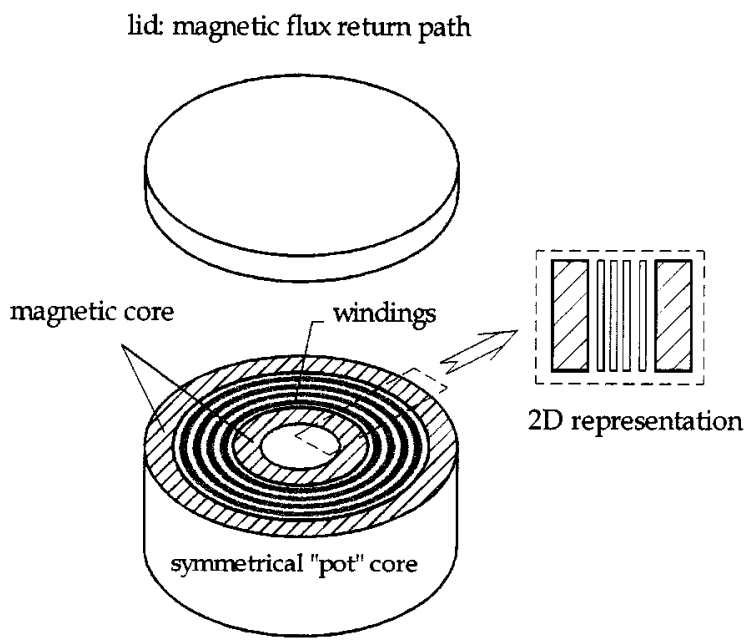

Fig. 17. 2-D cross section on axisymmetrical magnetic component: (a) inductor and (b) transformer.

to consider when designing for high-power densities. Another aspect entails the way that energy is collected and the length of the transport paths during the power conversion process. A typical capacitor comprises many layers of metallized dielectric film that are all connected in parallel. In Fig. 16, it is illustrated how each layer contributes an equal amount of energy flux in the $x$ direction, while the conductor that connects them in parallel serves to guide the collection of the energy resulting in the energy flux increasing in steps in the $y$ direction. The flow of electromagnetic energy associated with electrical charge is indicated with a clear arrow in this and other diagrams.

\section{Magnetic Components}

If the one-dimensional analysis of magnetic-induced energy flux is applied to a magnetic component with rotational symmetry a fairly good description of a real 3-D structure is achieved. A pot-core resembles such a magnetic component and is illustrated in Fig. 17. In order to simplify the geometry, the curvature is ignored and a 2-D representation, as shown in the same figure, is used in the subsequent discus-

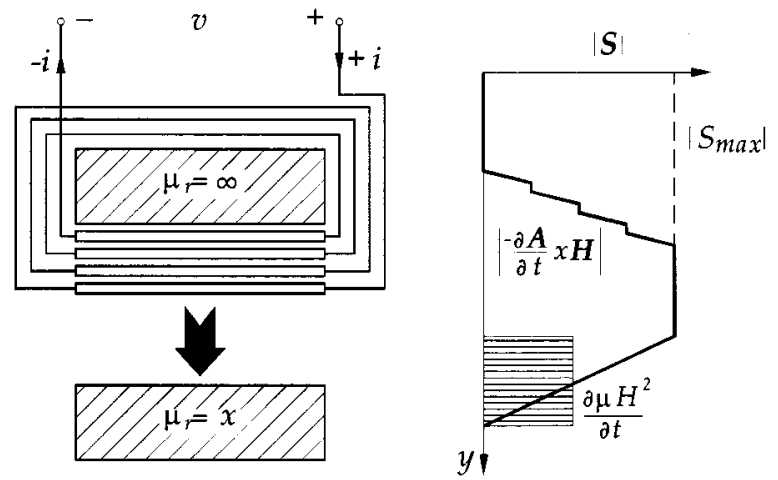

(a)

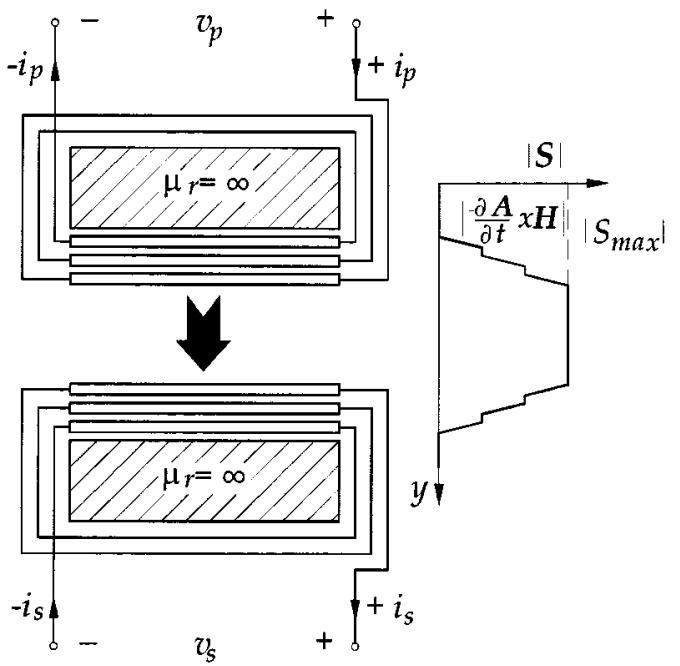

(b)

Fig. 18. 2-D magnetic components (a black arrow indicates exchange of magnetically induced electromagnetic energy): (a) inductor and (b) transformer.

sion. This presentation is essentially a top view of Fig. 6 including a flux return path and a possible secondary winding on the right.

If the magnetic limb adjacent to the winding terminals has an infinitely large permeability it will store no energy. If the second limb has a given permeability, with value " $x$," magnetic energy will be stored in the limb and we get an inductor. The top diagram in Fig. 18 shows this arrangement. The magnetic-induced energy flux is built up in steps by each winding layer as illustrated on the adjacent graph. The maximum value is given by (16). The magnetic-induced energy flux feeds on the incoming charge associated energy flux that enters the component via the conductor terminals. The energy is converted to stored magnetic energy and the value of $S$ decreases linearly eventually reaching zero. The exchange of magnetically induced electromagnetic energy between the winding and core is indicated by a black arrow in Fig. 18(a).

Fig. 18(b) illustrates the working of a transformer from an energy flux point of view. The primary winding fulfills the same role as the inductor winding discussed above. The energy flux is built up stepwise to a maximum value. The secondary winding absorbs the energy in the same stepwise manner feeding it to the secondary circuit. 


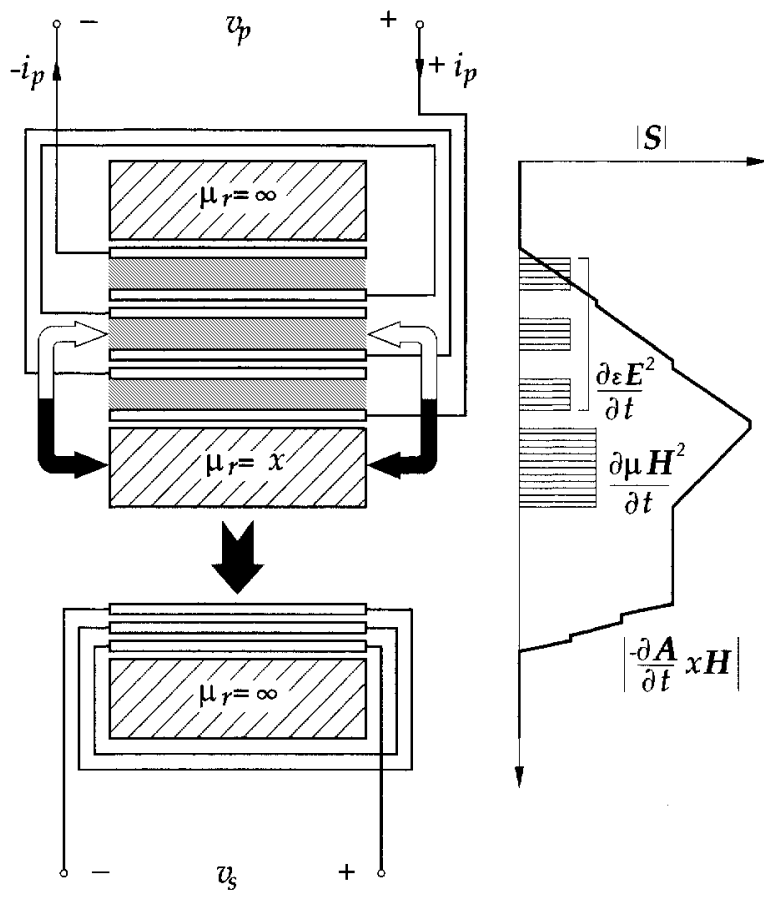

Fig. 19. Integrated and inductor-capacitor-transformer.

\section{Electromagnetically Integrated Components}

In filters and especially in the resonant tanks of soft switching converters, energy is transferred at high frequencies between capacitors and inductors. Due to the volume occupied by the packaging and due to the fact that these components have to be placed on crowded PCBs, one finds that distance between the reactive components is substantial. Apart from the obvious conduction losses the energy exchange also creates electromagnetic interference (EMI) problems. It is possible to integrate more than one electromagnetic energy storage function in one component, creating the possibility to improve the functionality and performance of passive components [16], [17]. A practical realization of this entails enhancing the interlayer capacitance in windings by placing a suitable dielectric between bifilar wound foil conductors and by tuning the reluctance between the primary and secondary winding by a magnetic core section with a low permeability [18], [19], as has been successfully illustrated in practical application [20].

Such an arrangement is shown in Fig. 19 and is essentially a composition of the single function components of Figs. 16 and 18. Electrical and magnetic energy storage are brought in close proximity to each other and the energy flux path length is minimized. On the graph on the right-hand side, the various energy components of (1) are illustrated spatially.

\section{Electromagnetic TRANSIENTS ON THE SWITCHES}

Modern semiconductor power switches have been continuously improved during the last three decades and the improved capability of these switches has been the main impetus behind new developments in power electronics in recent years. The breakdown voltage and current density of

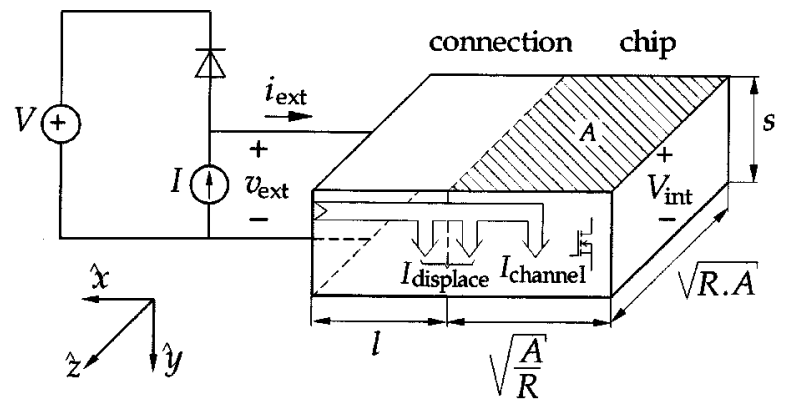

Fig. 20. Planar sandwich connection to power semiconductor chip.

the chips have been increasing steadily and the switching became faster. As a result, the circuit parasitics are playing a larger role in the circuit and oscillations between the stray inductance and the chip and structural capacitances are some of the main sources of RF-EMI in switch-mode converters.

Power semiconductor chips are mounted on a heat spreader and wire bonding is used to connect a number of parallel wires to the conductor leads in the case of conventional packages. In the case of power modules, hybrid integration is used and the chip is directly connected to the in-module copper circuit tracks with bonding wires. To increase current capability, a number of parallel bonding wires are often used, approaching a discretized planar conductor. Planar connections to the chips have recently been introduced, since this leads to advantages in interconnection technology [21], [22]. The assemblies of power semiconductors in the modules occupy a fair amount of surface due to heat spreading considerations. Consequently some distance exists between the chips and other components in the circuit. As a fundamental building block, Fig. 20 shows a planar connection to a chip allowing for a length $l$ to connect the chip to the rest of the circuit. The planar sandwich arrangement is representative of a future generation of low inductance transmission line packaging techniques and may present a preferred way of packaging. For the purpose of an electromagnetic analysis, MOSFETs as devices are placed between the conductor leads since for the present this allows a simpler analysis. Hard switching is assumed and the idealized "textbook"waveforms are shown in Fig. 21. During turnoff, the voltage $\left(v_{e x t}\right)$ rises linearly to the supply voltage, and then the diode takes over the current and the transistor current $\left(i_{e x t}\right)$ decreases linearly. At turnon, the opposite process takes place. The transistor must first commutate the full current before the voltage can change. The voltage and current therefore never change at the same time and this implies that the rate of field energy change involves always either of the terms in (1). When the current is switched, a voltage is induced internally in the package so that the voltage on the far side of the chip differs from the externally applied voltage. Similarly, the charge and discharging of the electric field between the planar conductors and the chip results in a displacement current ( $\left.I_{\text {displace }}\right)$, which adds or subtracts to the externally applied current to give the current that flows through the MOSFET channel $\left(i_{\text {channel }}\right)$. 


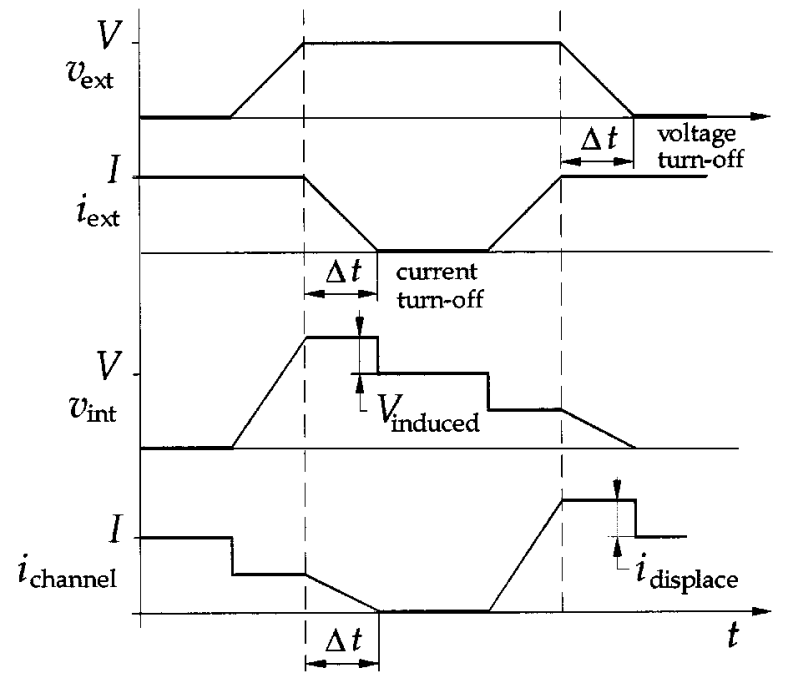

Fig. 21. Hard switching waveforms for the arrangement of Fig. 20.

\section{A. Current Turnoff}

It can be shown that the rate of change of the magnetic field in the chip is given by the following equations:

$$
\begin{array}{ll}
\frac{\partial H_{z}(x, t)}{\partial t}=-\frac{I}{\sqrt{R \cdot A} \Delta t} & \text { (connection) } \\
\frac{\partial H_{z}(x, t)}{\partial t}=-\frac{I \cdot x}{A \cdot \Delta t} & \text { (chip) }
\end{array}
$$

where

$I \quad$ value of the external current source;

$\Delta t \quad$ current fall (or rise) time;

A chip area;

$R \quad$ chip aspect ratio;

$x \quad$ position in the chip.

Faraday's law gives the following relationship between the induced electric field and the changing magnetic field:

$$
\frac{\partial E_{y}(x, t)}{\partial x}=-\mu_{0} \frac{\partial H_{z}(x, t)}{\partial t}
$$

and, using this equation the internally induced voltage, can readily be derived as

$$
V_{\text {induced }}=\frac{\mu_{0} I \cdot s}{\sqrt{R \cdot A} \Delta t}\left[1+\frac{1}{2} \sqrt{\frac{A}{R}}\right]
$$

where $s$ is the height of the planar connection.

The induced voltage adds to the externally applied voltage, thus creating the possibility of avalanche breakdown of the MOSFET at the point furthest removed from the circuit connection. At turnon, the induced voltage is subtracted and the chip voltage becomes smaller.

\section{B. Displacement Current}

Applying Amperé's law gives the following relationship between the magnetic field and the time varying electric field during switching:

$$
\frac{\partial H_{z}(x, t)}{\partial x}=J_{y}(x, t)+\varepsilon_{0} \varepsilon_{r} \frac{\partial E_{y}(x, t)}{\partial t} .
$$

Table 1

Switch Parameters

\begin{tabular}{c|c|c|l}
\hline parameter & $\underline{\text { low voltage chip }}$ & high voltage chip & description \\
\hline $\mathrm{A}$ & $1 \mathrm{~cm}^{2}$ & $1 \mathrm{~cm}^{2}$ & chip area \\
\hline $\mathrm{s}$ & $1 \mathrm{~mm}$ & $1 \mathrm{~mm}$ & $\begin{array}{l}\text { height of chip planar } \\
\text { connection }\end{array}$ \\
\hline $\mathrm{I}$ & $1 \mathrm{~cm}$ & $1 \mathrm{~cm}$ & length of planar connection \\
\hline $\mathrm{I}$ & $100 \mathrm{~A}$ & 5 & load current \\
\hline $\mathrm{V}$ & $50 \mathrm{~V}$ & 300 & supply voltage \\
\hline $\mathrm{C}_{\text {chip }}$ & $5 \mathrm{nF}$ & $0.5 \mathrm{nF}$ & chip capacitance \\
\hline
\end{tabular}

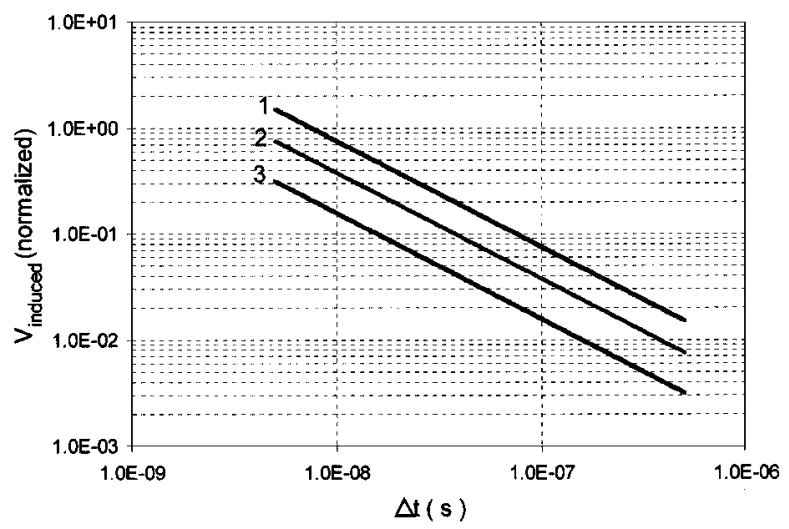

Fig. 22. Normalized induced voltage drop of low voltage device (normalized with respect to supply voltage): 1 -square chip with double current density, 2-square chip, and 3-rectangular chip, $1: 4$.

From this relationship, the displacement current can be derived as

$$
I_{\text {displace }}=\frac{\varepsilon_{0} V}{s \cdot \Delta t}\left[\varepsilon_{\text {connect }} l \sqrt{R \cdot A}+\varepsilon_{\text {chip }} A\right]
$$

where the equivalent relative permittivity of the chip is expressed in terms of the drain source capacitance, assumed to be voltage-independent

$$
\varepsilon_{\text {chip }}=\frac{C_{\text {chip }} A}{\varepsilon_{0}} .
$$

At turnoff, the displacement current adds to the existing current, increasing the current stress on the MOSFET channels. At turnon, the situation is reversed and the displacement current subtracts from the externally applied current.

\section{Stresses on Semiconductor Device due to Electromagnetic Transients}

The induced voltage due to the changing internal magnetic field is most pronounced in low-voltage transistors while the displacement current plays the prominent role at higher voltage ratings of transistors. Table 1 indicates the parameters that have been used to calculate some representative results on the stresses caused by the electromagnetic field transients using (20) and (22). The switching time $\Delta t$ is varied from 5 through $500 \mathrm{~ns}$, which is typical of the switching times possible with modern MOSFETs. In Figs. 22 and 23, graphs are shown of the normalized stresses 


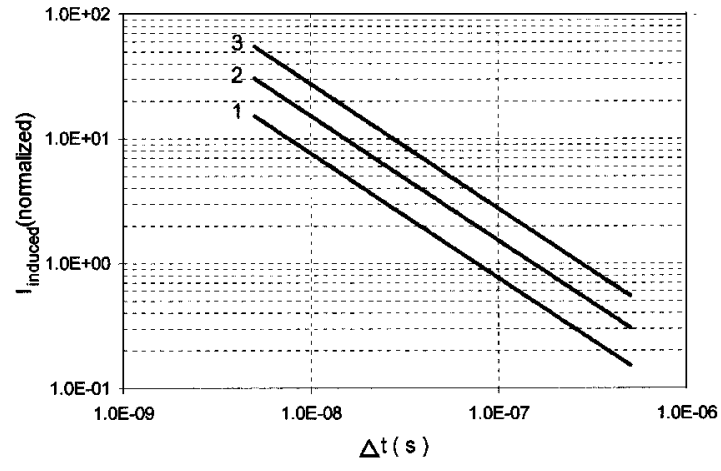

Fig. 23. Normalized displacement current of a high voltage device (nomenclature same as Fig. 23). (Normalized with respect to load current.)

imposed on the MOSFET chip by the changing electromagnetic fields during the switching transients. Three graphs are shown per figure. In the first one, a square chip using the current densities indicated in Table 1 is used (curve 2). In the second, the aspect ratio of the chip is changed from $1: 1$ to $1: 4$, (curve 3 ) while in the third the current density is doubled (curve 1) anticipating a technological improvement we could expect during the next couple of years. In Fig. 22, the parameters of the low-voltage MOSFET is used and the induced voltage is normalized with respect to the source voltage. In Fig. 23, the parameters of the high-voltage MOSFET is used and the displacement current is normalized with respect to the load current.

This analysis clearly shows that future limits to power semiconductor operation are electromagnetic. The losses and stresses measured externally are not representative of what the chip is subjected internally due to the electromagnetic transients. The value of the internally induced voltage is not only comparable to the external load and source voltages but may even exceed them. The induced over-voltage can be reduced if the aspect ratio of the chip is changed. Rectangular chips should be considered for low-voltage high-current devices where relative value of induced voltage is the largest. The displacement current is very significant in the example geometry that is presented, as can be seen from Fig. 23. The additional losses due to the increased channel current should be kept in mind during the thermal design.

\section{Electromagnetic Energy Propagation in A CONVERTER}

In Fig. 24, the circuit diagram of a full bridge series resonant converter is shown as an example. It comprises dc filters at the input and output, a MOSFET inverter, and a series resonant tank on the primary side of a transformer and a rectifier bridge on the secondary side. Using the 2-D representation applied to the various components discussed previously, the electromagnetic energy propagation in the converter can be sketched as indicated. In this electromagnetic flow picture, the semiconductors chips play a small role and are indicated by small rectangles. The two types of electromagnetic energy fluxes, namely the charge associated and the magnetic induced are given by Poynting vectors, indicated by clear and black arrows, respectively.

The electromagnetic power that enters the converter from the left is essentially constant due to the filtering action of $L_{i}$ and $C_{i}$. The switching action of the inverter causes a pulsating energy flux, which is buffered by the energy storage as the inductor and capacitor. It is worthwhile to note that, despite the inversion of voltage and current that takes place in the inverter, the energy flow is the same on the left and the right of the inverter. The frequency at which the transistors is switched is close to the resonant frequency of $L_{r}$ and $C_{r}$ and consequently energy oscillates between magnetic and electric field storage. This oscillation also serves to control the flow of energy to the output. The impedance posed by the resonant tank is strongly dependent on frequency and control is achieved by modulating the switching frequency. Then as the energy flows through the transformer, it changes from a charge associated energy flux to a magnetically induced flux. The four switches in the diode bridge does not physically affect the pulsating flow of energy, but allows the currents associated with the charge associated energy flux to flow in the appropriate direction by spatial change of the interconnection between input and output conductors. The electromagnetic energy storage in the output filter evens out the pulsating energy flow.

In Fig. 25, the input and output filters have been integrated, and electromagnetic integration has also been applied to the resonant tank and transformer. A neater picture emerges and the energy flow is consolidated and simplified. The regions where electric field and magnetic field energy are respectively stored, are placed in closer proximity to each other, making it easier to contain energy flux pulsations in smaller volumes. More flexibility is introduced to optimize the paths along which the electromagnetic energy flows.

\section{TOWARD ELECTROMAGNETIC INTEGRATION}

In this contribution rigorous, first-order modeling has been used to investigate electromagnetic energy propagation and establishes the demands that could be imposed by electromagnetic issues on current and future generations of power electronics technology. Four important aspects are now highlighted in Sections IX-AIX-D, leading to concluding observations on electromagnetic integration in Section IX-E.

\section{A. Packaging Requirements at High Switching Frequencies}

The charge-related electromagnetic energy flux density decreases at increasing frequency. This means that the cross section area of circuit interconnections becomes larger in converters that operate at high switching frequencies. The electric and magnetic energy storage exchange capability increases with frequency, so that at the same time the volume needed for the energy storage functions is reduced. One could therefore expect that the conductor interconnects will occupy a larger percentage of the volume of the future converter. 


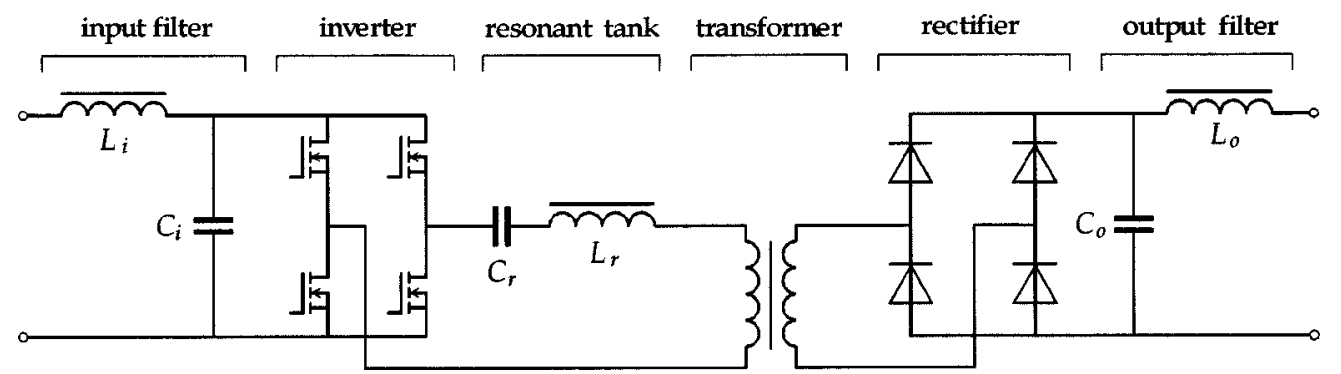

(a)

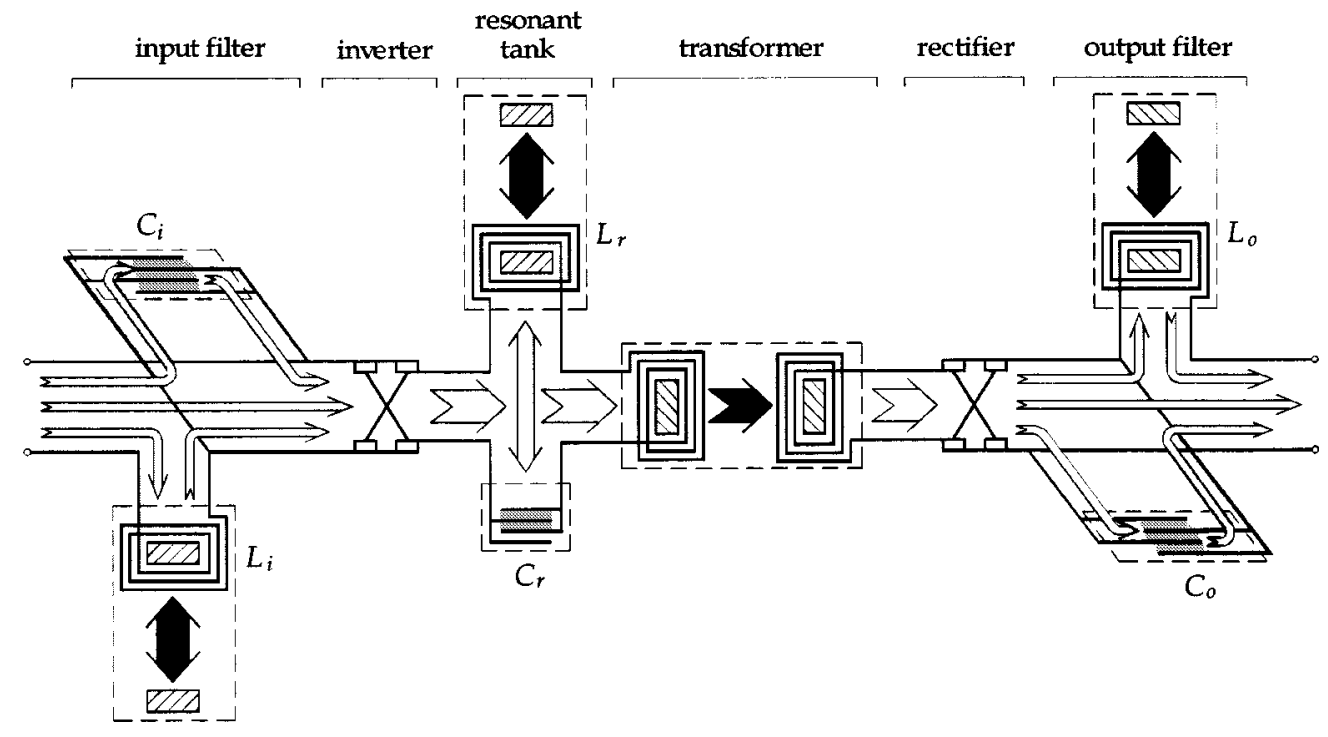

(b)

Fig. 24. Energy flow in a conventional resonant converter: (a) full bridge resonant converter and (b) electromagnetic energy propagation and exchange in the converter.

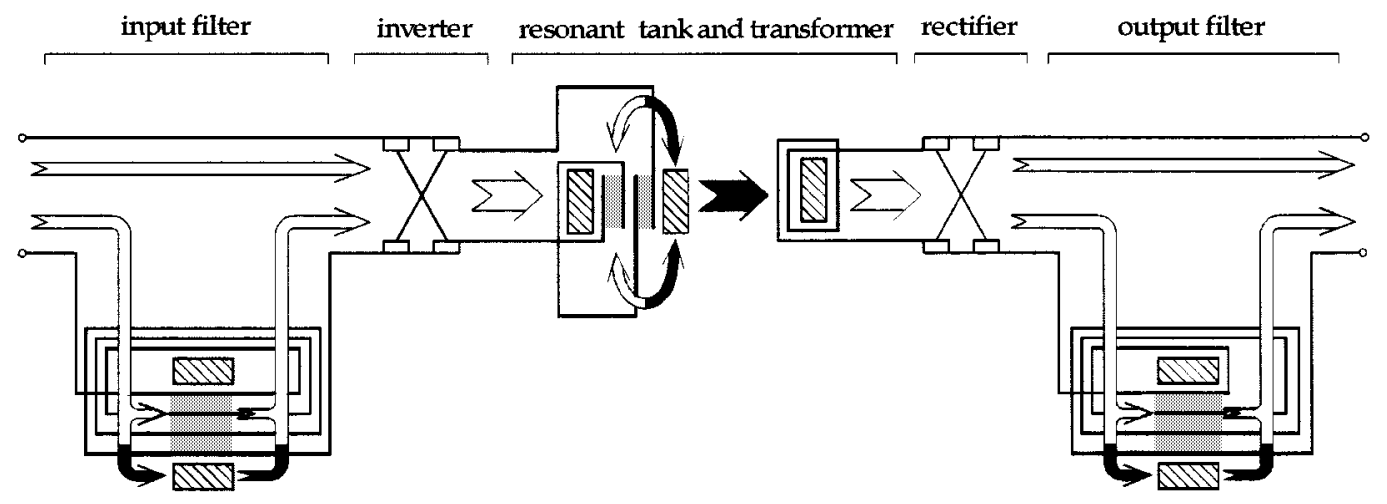

Fig. 25. Energy flow in an integrated resonant converter.

\section{B. Circuit Topologies at High Switching Frequencies}

Electrical field energy storage scales very well with frequency, resulting in very high energy exchange densities. Magnetic energy storage also benefits from frequency scaling but the improvement in energy exchange density is less and is also plagued by construction technology issues. It is therefore better to select circuit topologies that make more use of electric field energy storage and less of magnetic field energy storage.

\section{Switching Limitations}

The volume occupied by semiconductor switch assemblies is to a great extent determined by the heat spreading requirements. This means that the connections to the semiconductor chips need to be a certain length. The analysis presented in this paper indicated that electromagnetic effects cause considerable stresses in the current generation of semiconductor switches. This aspect deserves more attention and it will be better to restrict the switching speed and concentrate on reducing the conduction losses of next generations of switches. 
This will ease heat spreading requirements, leading to less electromagnetic volume of switching arrangements.

\section{Microfabricated Magnetic Components}

For high-power densities in magnetic components, a large value of the magnetic vector potential is required. A general limitation of microfabrication technologies are that the thickness of the magnetic material layers are small. The analyses show clearly that this leads to very low values of energy flux when compared to conventional magnetic cores. New approaches or alternatives to microfabrication are needed when dealing with magnetic components.

\section{E. The Concept of Electromagnetic Integration}

Electromagnetic integration implies the spatial arrangement of the switching function, the conduction function and the electromagnetic energy storage function (see Fig. 2) in such a way that optimal energy propagation through the converter and optimal electromagnetic transient conditions for the switches and the materials used are achieved. Although this presents an enormous challenge, some of the previously presented analysis and discussion already gives some indication of what can be achieved, such as:

- location of electric and magnetic energy storage in the same volume or interleaved volume, provides a means to reduce interconnections, thus reducing volume as frequency increases;

- electromagnetic volume reduction in switching arrangements will reduce limiting stresses;

- for constant frequency, losses in materials increase with spatial increasing energy flux density-the converter should therefore be configured to encourage electromagnetic energy propagation as uniformly as possible;

- planar forms of electromagnetic structures (capacitors, transformers, semiconductors) tend to enhance uniform electromagnetic energy propagation.

However, to evaluate the possibilities of electromagnetic integration in power electronic converters properly, much more in-depth electromagnetic analysis than presented in this paper is necessary.

\section{CONClusion}

The shrinking dimensions and increasing frequencies in power electronics technology have brought about a realization of the limitations of circuit theory and the importance of the electromagnetic nature of the converter. An electromagnetic approach has been presented that does not use the concepts of circuit elements $R, L$, and $C$. As a first approach, the propagation of electromagnetic energy through the converter by using the Poynting vector concept for analysis of energy flux in components has been described. Fundamental limitations and frequency scaling trends based on the material properties and geometric aspects have been identified and calculated. These give some indication of where the electromagnetic aspects of power conversion will take us in the future. It is also illustrated that, although the semiconductor power switches handle very little energy flow, the electromagnetic nature of the switching itself is an eventual limiting phenomenon in terms of internal transients. Although electromagnetic integration holds out the possibility of improving future high-frequency high-density electronic power processing, much work still needs to be done to formalize this approach.

\section{REFERENCES}

[1] T. G. Wilson, Sr., "The evolution of power electronics," IEEE Trans. Power Electron., vol. 15, pp. 439-446, May 2000.

[2] J. D. van Wyk, H. C. Skudelny, and A. Mueller-Hellman, "Power electronics, control of the electromechanical conversion process and some applications," Proc. Inst. Elect. Eng., pt. B, vol. 133, pp. 369-399, Nov. 1986.

[3] T. G. Wilson, Jr., "Life after the schematic: The impact of circuit operation on the physical realization of electronic power supplies," Proc. IEEE, vol. 76, pp. 325-334, Apr. 1998.

[4] J. A. Ferreira, "Engineering science considerations for integration and packaging," in Conf. Rec., IEEE PESC 2000 (31st Annu. Power Electronics Specialists Conf.), vol. 1, Galway, Ireland, June 2000, pp. $12-18$.

[5] J. D. van Wyk and F. C. Lee, "Power electronics at the dawn of the new millennium - Status and future," in Conf. Rec., IEEE PESC '99 (30th Annu. Power Electronics Specialists Conf.), vol. 1, Charleston, SC, July 1999, pp. 3-12.

[6] O. Heaviside, Electrical Papers. New York: Macmillan, 1892, p. 438.

[7] J. H. Poynting, "On the transfer of energy in the electromagnetic field," Philos. Trans. R. Soc., vol. 175, p. 353, 1884.

[8] J. J. Thomson, Recent Researches in Electricity and Magnetism. London: Claredon, 1893, p. 280.

[9] J. Slepian, "Energy and energy flow in the electromagnetic field," Trans. Amer. Inst. Elect. Eng., vol. 13, p. 277, 1942.

[10] R. P. Feynman, R. B. Leighton, and M. L. Sands, The Feynman Lectures on Physics. Reading, MA: Addison-Wesley, 1964, vol. II, pp. 17-6-27-11.

[11] P. Hammond, "Electromagnetic energy transfer," Inst. Elect. Eng. Monograph 286, Feb. 1958.

[12] J. A. Ferreira, "Application of the Poynting vector for power conditioning and conversion," IEEE Trans. Educ., vol. 31, p. 257, Nov. 1988.

[13] J. Slepian, "The flow of power in electrical machines," Elec. J., vol. 16, p. 303, 1919.

[14] P. Dowell, "Effects of eddy currents in transformer windings," Proc. Inst. Elect. Eng., vol. 113, p. 1387, Aug. 1966.

[15] J. A. Ferreira, "Analytical computation of AC resistance of round and rectangular litz wire windings," Proc. Inst. Elect. Eng., pt. B, vol. 139, pp. 21-25, Jan. 1992.

[16] M. C. Smit, J. A. Ferreira, and J. D. van Wyk, "Application of transmission line principles to high frequency power converters," in IEEE Power Electronics Specialists Conf. Rec. (PESC-92), vol. 2, June-July 1992, pp. 1423-1430.

[17] J. D. van Wyk and J. A. Ferreira, "Some present and future trends in power electronic converters," in Proc. IEEE Int. Conf. Industrial Electronics, Control, Instrumentation and Automation, IECON, vol. 1, Nov. 1992, pp. 9-18.

[18] J. A. Ferreira, I. W. Hofsaier, and J. D. van Wyk, "Exploiting the third dimension in power electronics packaging," in Proc. IEEE APEC, 1997, pp. 419-423.

[19] I. W. Hofsajer, J. A. Ferreira, and J. D. van Wyk, "A new manufacturing and packaging technology for the integration of power electronics," in Conf. Rec. IEEE IAS, vol. 1, 1995, pp. 891-897.

[20] J. T. Strydom, J. D. van Wyk, and J. A. Ferreira, "Some limits of integrated LCT-modules for resonant converters at $1 \mathrm{MHz}$," IEEE Trans. Ind. Applicat., vol. 3, pp. 820-828, May/June 2001.

[21] S. Haque, K. Xing, R.-L. Lin, C. Suchicital, G.-Q. Lu, D. J. Nelson, D. Borojevic, and F. C. Lee, "An innovative technique for packaging power electronic building blocks using metal posts interconnected parallel plate structures," IEEE Trans. Adv. Packag., vol. 22, pp. 136-144, 1999.

[22] X. Liu, S. Haque, and G.-Q. Lu, "Three-dimensional flip-chip on flex packaging for power electronics applications," IEEE Trans. Adv. Packag., to be published. 


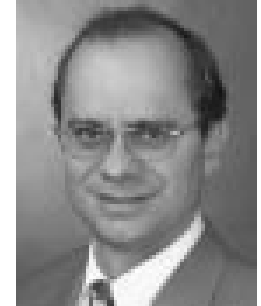

Jan Abraham Ferreira (Member, IEEE) was born in Pretoria, South Africa. He received the B.Sc. Eng., M.Sc. Eng., and Ph.D. degrees in electrical engineering from the Rand Afrikaans University, Johannesburg, South Africa, in 1980, 1982 , and 1988, respectively.

He worked in industry at ESD (Pty), Ltd. from 1982 to 1985 . From 1986 until 1997, he was at the Faculty of Engineering, Rand Afrikaans University, where he held the Carl and Emily Fuchs Chair of Power Electronics. Since 1998, he has been Professor at the ITS Faculty of the Delft University of Technology, The Netherlands.

Prof. Ferreira is the Transactions Review Chairman of the IEEE IAS Power Electronic Devices and Components committee, chairman of the IEEE Joint IAS/PELS Benelux chapter and chairman of the CIGRE SC14 national committee of the Netherlands. He is also a member of the IEEE PESC Adcom, and a member of the executive committee of the EPE Society.

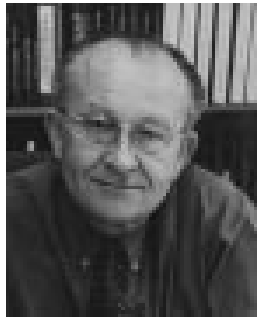

J. Daan van Wyk (Fellow, IEEE) received the M.Sc. Eng. degree from the University of Pretoria, South Africa, in 1966, the Doctor of Technical Science degree from the University of Technology, Eindhoven, The Netherlands, in 1969, and the D.Sc. (Eng.) degree (honoris causa) from the University of Natal, South Africa, in 1996.

He was with the S. A. Iron and Steel Corporation, the University of Pretoria, and the technical and scientific staff of the University in Eindhoven, between 1961 and 1971. From 1971 to 1995, he was a chaired Professor of Electrical and Electronic Engineering at the Rand Afrikaans University, Johannesburg, holding Chairs in Electronics and in Power Electronics until 1992. He founded the Industrial Electronics Technology Research Group in the Faculty of Engineering in 1978 and directed this unit until 1999. Since July 1995, he held the special University Council Research Chair in Industrial Electronics at the Rand Afrikaans University and from 2000 onward holds this in a part-time capacity. He joined the Bradley Department of Electrical and Computer Engineering in January 2000, where he is the J. Byron Maupin Professor of Engineering at Virginia Tech, Blacksburg, VA, working in the National Science Foundation Engineering Research Center for Power Electronics Systems. He has authored or co-authored more than 300 publications

Dr. van Wyk has been co-recipient of ten IEEE prize paper awards for some of this work, and was the recipient of the prestigious IEEE William E. Newell Power Electronics Award in 1995 and an IEEE Third Millennium Medal in 2000. He is active in several capacities within the IEEE and its Societies. He has received a range of other awards from IEEE Societies as well as from the South African Institute of Electrical Engineers. He is a fellow of the South African Institute of Electrical Engineers. 